\title{
Homologación lingüística nacional del Dizziness Handicap Inventory (test de discapacidad vestibular)
}

\author{
National linguistic homologation of the Dizziness Handicap Inventory \\ Alejandro Peña $\mathbf{M}^{\mathbf{1}}$.
}

\begin{abstract}
RESUMEN
La sintomatología de los síndromes vertiginosos es mayoritariamente subjetiva, de difícil evaluación para el médico. En tal sentido es útil contar con elementos que permitan estandarizar los síntomas que el paciente sufre y poder así medir el grado de discapacidad que la enfermedad le pueda provocar. Con este propósito, hace ya algunos años, Jacobson y Newman publicaron el llamado test del Dizziness Handicap Inventory (Test de discapacidad vestibular). Dicho test ha sido posteriormente validado por numerosos estudios, otorgándole alta veracidad y confiabilidad estadística. El presente estudio traduce al español cotidiano de los chilenos este test, para tener una herramienta confiable, que permita al otorrinolaringólogo poder con mayor seguridad, determinar el grado de discapacidad sea por desequilibrio o mareos que un síndrome vertiginoso le pueda provocar a un paciente.
\end{abstract}

Palabras clave: Dizziness Handicap Inventory, Discapacidad vestibular.

\begin{abstract}
The symptoms of dizziness syndromes are mainly subjective and difficult to evaluate by the physician. It is thus highly useful to count on elements that allow standardization of the patient's symptoms, and to have an available measurement of vestibular disability the disease may cause. In order to achieve this, Jacobson and Newman published some time ago the so called Dizziness Handicap Inventory. This test has been later validated by several studies, which gave it high veracity and statistical reliability. This paper translates the test into everyday chilean spanish in order to have a reliable tool that allows an ENT specialist to determine the patient's degree of discapacity.
\end{abstract}

Key word: Dizziness handicap inventory, Vestibular disability.

10torrinolaringólogo, Facultad de Medicina, Universidad Católica del Maule. 


\section{INTRODUCCIÓN}

La sintomatología de los síndromes vertiginosos es principalmente subjetiva, por tal motivo es difícil la evaluación clínica de estos pacientes. Esta dificultad es mayor cuando el médico se enfrenta a algunos de los siguientes problemas, primero: cuando trata de objetivar los resultados terapéuticos farmacológicos obtenidos en estos pacientes y conocer el progreso en su mejoría; segundo: cuando desea evaluar estadísticamente el éxito logrado en aquellos pacientes aquejados de un síndrome vertiginoso prolongado, a quienes se les ha indicado una terapia de rehabilitación vestibular, particularmente cuando estos estudios se efectúan con un grupo control; tercero: cuando desea conocer qué criterios usar, para indicar la prolongación de una licencia médica, de un paciente que dice continuar mareado 0 sentirse permanentemente desequilibrado e incapacitado para trabajar, y cuarto: cuando acude a su consulta, un paciente que sufre de un síndrome vertiginoso por lesión laberíntica bilateral quien después de seis meses, dice persistir mareado y solicita su invalidez laboral ¿qué pautas deben seguirse para calificar esta posible discapacidad? Respecto a este último punto la comisión técnica de la Superintendencia de AFP, publica un documento sobre el menoscabo global de la persona por impedimentos vertiginosos, con pautas muy vagas 0 imprecisas que pueden prestarse a confusión ${ }^{1}$.

\section{Test de discapacidad vestibular}

Frente a este problema universal, que es el poder evaluar los síntomas que provocan una discapacidad por disfunción vestibular y en que las técnicas o procedimientos convencionales del examen otoneurológico, son inadecuados para cuantificar el impacto del mareo o de la sensación de desequilibrio que en la vida diaria pueden experimentar estos pacientes, hace ya algunos años Jacobson, y Newman publicaron un trabajo², proponiendo una encuesta, el Dizziness Handicap Inventory o test de discapacidad vestibular, que debe ser respondida por los propios enfermos considerando los factores, funcionales, físicos y emocionales involucrados en el cuadro clínico.
Esta encuesta la aplicaron en 106 pacientes, efectuando un prolijo estudio estadístico que demostró su confiabilidad. Numerosos estudios posteriores, respaldados por su aplicación en gran cantidad de enfermos de varios países, han confirmado la utilidad de este test, y su confiabilidad estadística ${ }^{3-7}$, ratificando el estudio original y que demuestran que este test, tiene un buen nivel de exactitud y confianza, recomendándose como una medida de la discapacidad en aquellos pacientes con mareos e inestabilidad8.

Este test ha sido traducido a varios idiomas y adecuado lingüísticamente según el léxico de cada nación; existe una versión española del Dizziness Handicap Inventory ${ }^{3}$ o test de discapacidad vestibular, pero que no es aplicable en Chile, pues aunque hablamos el mismo idioma, el lenguaje y expresiones cotidianas son diferentes al de España, razón por la cual, en este trabajo se publica una versión chilena con un lenguaje simple y comprensible para la mayor parte de la población que otorga una mayor confiabilidad en las respuestas de los pacientes.

\section{Dizziness Handicap Inventory (Test de Discapacidad Vestibular)}

Este test consta de 25 preguntas que deben ser respondidas por el propio paciente lo que habitualmente no toma más de 10 minutos. Comprende dicho test tres tipos de preguntas que abarcan los problemas emocionales, físicos y funcionales que pueda experimentar el paciente con un síndrome vertiginoso no compensado. Frente a cada pregunta el paciente tiene tres opciones de respuesta: siempre otorgándosele 4 puntos a estas respuestas, a veces, otorgándole 2 puntos a estas respuestas, y nunca, no otorgándoles punto a estas respuestas, de tal manera que el puntaje máximo obtenible es 100. Como parece obvio, a mayor puntaje es mayor el grado de discapacidad del paciente, considerando siempre como variable importante la edad, sobre todo al comparar resultados con otros pacientes.

Referente a este último punto, un estudio francés de respuestas de este test en 120 pacientes normales de entre 20 y 79 años, reveló que existe un aumento significativo del puntaje del test cuando se aplica en pacientes de mayor edad ${ }^{9}$. 


\section{Test del Dizziness Handicap Inventory, o de discapacidad vestibular, homologación lingüística nacional}

1. ¿Cúando usted mira hacia arriba se siente más mareado (a) 0, aumenta su problema? FI

2. ¿Debido a su problema 0 , mareo ¿se siente como fracasado (a)? $\mathbf{E}$

3.- ¿Debido a su mareo o problema ¿evita hacer viajes de placer 0 hacer alguna diligencia? $\mathbf{F}$

4. Cuando camina por los pasillos de un supermercado ¿siente que aumenta su problema o el mareo? FI

5. ¿A causa de su problema, o del mareo le cuesta acostarse o levantarse de la cama? $\mathbf{F}$

6. ¿Debido a su problema o el mareo, trata de participar lo menos posible en actividades tales como, salir a comer afuera, 0 ir al teatro, 0 ir a fiestas, 0 salir a bailar? $\mathbf{F}$

7. ¿A causa de su problema o mareo le cuesta leer? $\mathbf{F}$

8. ¿Al tener que realizar actividades más exigentes como algún deporte 0 , trabajos pesados de la casa (barrer, limpiar pisos, guardar los platos) siente que aumenta su problema o su mareo? FI

9. ¿Debido a su problema o por el mareo tiene miedo de salir sola (0) de su casa sin que nadie la acompañe? E

10. ¿A causa de su problema o mareo se siente incómoda (0) frente a otras personas? $\mathbf{E}$

11. ¿Al hacer movimientos rápidos de su cabeza nota que aumenta su problema o mareo? FI

12. ¿Debido a su problema o mareo evita las alturas, como por ejemplo tener que subir a un edificio de varios pisos? $\mathbf{F}$

13. ¿Al darse vuelta en la cama siente que aumenta su problema 0 el mareo? FI

14. ¿Debido a su problema o mareo le cuesta hacer los trabajos pesados de la casa 0 del patio? $\mathbf{F}$

15. ¿Debido a su problema o mareo se avergüenza al pensar que la gente crea que pueda estar borracho (a), o drogado (a)? $\mathbf{E}$

16. ¿A consecuencias de su problema o mareo le cuesta caminar solo (a)? $\mathbf{F}$

17. ¿Al bajar de la vereda a la calle 0 calzada aumenta su problema o mareo? FI

18. ¿Debido a su problema o mareo le cuesta concentrarse? $\mathbf{E}$

19. ¿Debido a su problema o mareo le cuesta caminar en la noche a oscuras dentro de su casa? $\mathbf{F}$

20. ¿A consecuencias de su problema o mareo tiene miedo de quedarse solo (a), en su casa? $\mathbf{E}$

21. ¿Debido a su problema o mareo se siente incapaz 0 inútil? $\mathbf{E}$

22. ¿A consecuencias de su problema o mareo ha tenido dificultades de relaciones personales con sus parientes 0 amigos? $\mathbf{E}$

23. ¿Debido a su problema o mareo se encuentra que está deprimido (a) 0 entristecido (a)? E

24. ¿El problema que usted tiene 0 el mareo que siente han interferido con su trabajo o responsabilidades familiares? $\mathbf{F}$

25. ¿Al agacharse 0 inclinarse hacia delante como por ejemplo al recoger un objeto del suelo siente que aumenta su problema o su mareo? FI

Tipos de respuesta: Siempre 4 puntos. A veces 2 puntos. Nunca 0 punto.

Subdivisión del test: Categorías de preguntas.

Aspecto emocional E (9): 2, 9, 10, 15, 18, 20, 21, 22, 23.

Aspecto funcional $F(9): 3,5,6,7,12,14,16,19,24$.

Aspectos físicos FI (7): 1, 4, 8, 11, 13, 17, 25.

Subpuntaje emocional máximo: 36 puntos

Subpuntaje funcional máximo: 36 puntos

Subpuntaje físico máximo: 28 puntos 


\section{BIBLIOGRAFÍA}

1. Normas para la evaluación y calificación del grado de invalidez de los trabajadores afiliados al nuevo sistema. Superintendencia de AFP. $5^{\text {a }}$ Edición 2007.

2. Jacobson Gary, Newman Gary. The development of the Dizziness Handicap Inventory. Arch Otolaryngol Head Neck Sur 1990; 116(4): 424-7.

3. Ceballos Ricardo, Vargas Alejandro. Aplicación y utilidad del Dizziness Handicap Inventory en pacientes con vértigo del Servicio de Otorrinolaringología del Hospital de Especialidades del Centro Médico Nacional Siglo XXI. An Med Asoc Med Hosp ABC 2004; 49 (4): 176-83.

4. Castro as, Gazzola JM, Natur J, Gananza FF. Brasilian versión of the Dizziness Handicap Inventory. Pro Fono 2007; 19 (1): 97-104.

5. Nola G, Mostardini C, Salvi V, Ercolani A, Ralli G. Validity of Italian adaptation of The Dizziness Handicap Inventory and evaluation of the quality of life in patients with acute dizziness. Acta Otorhinolaryngol Ital 2010; 30: 190-7.

6. Kurre Annette, Bastiaenen Caroline, Jaw van Gool Christel. Exploratory factor analysis of the Dizziness Handicap Inventory (German version). BMC Ear, Nose and Throat disorders 2010; $10: 3$.

7. Kurre Annette, Bastiaenen Caroline, Straumann D. Translation, cross-cultural adaptation and reliability of german version of the dizziness handicap inventory. Otol Neurotol 2009; 30(3): 359-67.

8. Tamber AL, Wilhelmesen KT, Strand LI. Measurement properties of the Dizziness Handicap Inventory by cross-sectional and longitudinal designs. Health Qual Life Outcomes 2009; 7: 101-10.

9. Nyabenda A, Briart C, Deggouj N, Gersdorff M. Étude normative et de la reproductibilité d'une échele du handicap lié aux troubles de l'equilibre et aux vertiges."Dizziness Handicap Inventory", version francaise. Ann de réad et méd phy 2004; 47 (3): 105-13. 